\title{
CircWHSC1 promotes ovarian cancer progression by regulating MUC1 and hTERT through sponging miR-145 and miR-1182
}

\author{
Zhi-Hong Zong ${ }^{1,2}$, Yu-Ping Du ${ }^{3}$, Xue Guan ${ }^{3}$, Shuo Chen ${ }^{1,2}$ and Yang Zhao ${ }^{1,2^{*}}$
}

\begin{abstract}
Background: Circular RNAs are key regulators in human cancers, however, there is a lack of studies on circRNAs' specific functions in ovarian cancer.

Methods: Our study used qRT-PCR to detect the differentially expressed circRNAs between normal ovaries and ovarian cancer tissues. Cell function experiments were performed to verify the role of overexpression and silence of circWHSC1, including MTT assay, cell apoptosis assay, wound healing and Matrigel-coated Transwell assay. In vivo tumorigenesis model was constructed by subcutaneous injection in nude mice. Bioinformatics analysis predicted the possible binding sites of circWHSC1 with miRNAs, and confirmed with dual-luciferase reporter assay and RNA pull-down assay. The exosomes were extracted with ultracentrifugation. HE staining was also used to detect morphology of nude mice peritoneum.
\end{abstract}

Results: We found that circWHSC1 was up-regulated in ovarian cancer tissues, and circWHSC1 expression was higher in moderate \& poor differentiation ovarian cancer tissues than in well differentiation ovarian cancer tissues. Overexpression of circWHSC1 increased cell proliferation, migration and invasion, and inhibited cell apoptosis. Silence of circWHSC1 exerted the opposite effects. Additionally, circWHSC1 could sponge miR-145 and miR-1182 and up-regulate the expression of downstream targets MUC1 and hTERT. Exosomal circWHSC1 can be transferred to peritoneal mesothelial cells and promotes peritoneal dissemination.

Conclusions: Our study demonstrates the highly expressed circWHSC1 in ovarian cancer promotes tumorigenesis by sponging miR-145 and miR-1182, and its exosome forms induce tumor metastasis through acting on peritoneal mesothelium.

Keywords: CircRNA, Ovarian cancer, Exosome, Tumorigenesis and progression

\section{Background}

As the most lethal gynecology malignancy worldwide, ovarian cancer accounts for $5 \%$ of female cancer deaths. In 2018, it is estimated that 22,240 new cases of ovarian cancer will be diagnosed in US $[1,2]$. The anatomical features of the ovarian position deep in the abdominal

\footnotetext{
*Correspondence: yida.zhaoyang@163.com

'Department of Gynecologic Oncology Research Office, The Third Affiliated Hospital of Guangzhou Medical University, No.63 Duobao Road, Liwan District, Guangzhou 510150, Guangdong, China

${ }^{2}$ Department of Obstetrics and Gynecology, Key Laboratory for Major Obstetric Diseases of Guangdong Province, and Key Laboratory of Reproduction and Genetics of Guangdong Higher Education Institute in Guangdong Province, The Third Affiliated Hospital of Guangzhou Medical University, Guangzhou 510150, China

Full list of author information is available at the end of the article
}

cavity give the clinical features of its late diagnosis and low survival rates. Therefore, research on the molecular biological mechanism of ovarian cancer tumorigenesis and progression has received extensive attention.

CircRNAs are reported to be associated with some diseases and function as potential biomarkers for diagnosis. In particular, its "microRNA sponge" effect in tumorigenesis has been widely studied by researchers [3]. Due to the covalently closed loop structure, circRNAs have high tolerance to RNaseR exonuclease and remain more stable than its homologous linear transcript [4]. Recent studies showed that circRNAs are stably abundant in exosomes. Exosomal circRNAs were intact and enriched

(c) The Author(s). 2019 Open Access This article is distributed under the terms of the Creative Commons Attribution 4.0 International License (http://creativecommons.org/licenses/by/4.0/), which permits unrestricted use, distribution, and reproduction in any medium, provided you give appropriate credit to the original author(s) and the source, provide a link to the Creative Commons license, and indicate if changes were made. The Creative Commons Public Domain Dedication waiver (http://creativecommons.org/publicdomain/zero/1.0/) applies to the data made available in this article, unless otherwise stated. 
by at least 2 -fold in exosomes compared to those in producer cells [5].

In this study, we have used gene chip technology to detect the presence of circular RNA in ovarian cancer in very few samples. We found that several circular RNAs including hsa_circ_0001387 (http://www.circbase.org/) are relatively highly expressed in ovarian cancer tissues and have not been reported in tumors. Circ_0001387 (from here on referred to as circWHSC1) is derived from exonic back-splicing of WHSC1 gene. We subsequently expanded the sample size and detected circWHSC1 expression and found that its expression level in ovarian cancer was higher than that in normal tissues. Therefore, we further explored underlying functions of circWHSC1 in ovarian cancer.

\section{Methods}

\section{Ovarian cancer specimens}

Seventy-nine epithelial ovarian carcinoma samples and 13 normal ovary samples were collected at the Department of Gynecology in First Affiliated Hospital of China Medical University (Shenyang, China). All the enrolled patients signed informed consents, and none of them had undergone chemotherapy or radiotherapy prior to surgery. Our study was approved by China Medical University Ethics Committee.

\section{Cell culture and transfection}

Human ovarian carcinoma cell lines CAOV3 and OVCAR3 were cultured in RPMI 1640 (HyClone, USA) supplemented with $100 \mathrm{U} / \mathrm{mL}$ of penicillin/streptomycin and $10 \%$ fetal bovine serum. The incubator was set at $37{ }^{\circ} \mathrm{C}$ with $5 \%$ $\mathrm{CO} 2$. Lipofectamine3000 was used to perform transfection according to manufacturer's instructions. The circWHSC1 lower-expressed cell line CAOV3 was stably transfected with pHBLV-CMV-circWHSC1 plasmid (Hanbio Biotech, Shanghai, China), while circWHSC1 higher-expressed cell line OVCAR3 was transfected with sh-circWHSC1-GFP targeting to destroy its circular structure. After transfection, both circWHSC1 overexpressed and down-regulated cell lines were further selected with $2 \mu \mathrm{g} / \mathrm{mL}$ puromycin. The sequences of circWHSC1 and shRNA can be found in Additional file 1: Table S3.

\section{MTT assay}

The circWHSC1 overexpressed and down-regulated cells with control group were counted and seeded in 96-well plates. $0 \mathrm{~h}, 24 \mathrm{~h}, 48 \mathrm{~h}$ and $72 \mathrm{~h}$ after seeding cells, $20 \mu \mathrm{L}$ of $5 \mathrm{mg} / \mathrm{mL}$ MTT solution (Solarbio, China) was added in each well, and incubated for $3 \mathrm{~h}$. After discarding the supernatant, $150 \mu \mathrm{L}$ of DMSO was added to dissolve formazan. Microplate spectrophotometer was used to detect the absorbance at $490 \mathrm{~nm}$.

\section{Cell apoptosis assay}

The cells were collected at $1500 \mathrm{r}$ for $5 \mathrm{~min}$ and washed with PBS. $5 \mu \mathrm{L}$ of 7AAD and PE-labeled Annexin V (BD Biosciences) were used to label apoptotic cells for each sample. The cells apoptosis rate was determined with flow cytometry.

\section{Wound healing assay}

$200-\mu \mathrm{L}$ pipette tip was used to scratch wounds when cell confluence reached $80 \%$. After washing with PBS, the cell culture medium was replaced with $20 \mu \mathrm{g} / \mathrm{ml}$ of mitomycin C in FBS-free medium, in order to weaken the interference effects of cell proliferation. The healing wounds were photographed twice at $0 \mathrm{~h}$ and $48 \mathrm{~h}$ after scratching.

\section{Transwell assay}

50,000 cells were seeded onto filters coated with Matrigel in each chamber. $200 \mu \mathrm{L}$ of serum-free medium was added at the upper compartment and $600 \mu \mathrm{L}$ of medium with 10\% FBS was added at bottom. After incubation for $48 \mathrm{~h}$, cells above the filter were wiped off with a cotton swab, while the invaded cells at bottom were fixed, then stained with crystal violet.

\section{RT-qPCR}

Total RNA from cells and tissues were extracted with TRIzol reagent. $5 \mu \mathrm{g}$ of the RNA was reverse transcribed to complementary DNA and amplified using SYBR Premix Ex Taq ${ }^{\text {Tx }}$ II kit (Takara, Shiga, Japan). The comparative expression level of circWHSC1was compared with $18 \mathrm{~S}$ with $2-\Delta \Delta \mathrm{Ct}$ method.

\section{Western blotting}

Total protein was extracted with RIPA buffer and boiled for degeneration. $30 \mu \mathrm{g}$ of the protein were loaded into 7.5\% SDS-PAGE. After being electrotransferred and blocking with $3 \% \mathrm{BSA}$, the membranes were incubated with primary antibodies. Rabbit against MUC1, CD63, E-cadherin, N-cadherin (1:1000; Proteintech, USA), hTERT (1:1000; Bioss, China), HSP70 (1:500; Boster, USA), TSG101 (1: 1000; ABclonal, USA), $\beta$-actin (1:3000; Proteintech, USA) and mouse against CD9 (1:1000; Proteintech, USA) were used as primary antibodies. Anti-rabbit or anti-mouse IgG conjugated with HRP (1:5000; Abbkine, USA) was used as the secondary antibody. Enhanced chemiluminescence (Abbkine, USA) was used to display the bands.

\section{In vivo xenograft tumor model}

4-week-old female BALB/c nude mice were subcutaneously or intraperitoneally injected with $1 \times 10^{7}$ CAOV3 cells. To explore the role of circWHSC1-rich exosomes, the nude mice were divided into 2 groups after tumorigenicity. One group was intraperitoneally injected with $200 \mu \mathrm{g}$ of exosomes 
isolated from circWHSC1-overexpressing CAOV3 cells, and the other group were injected with PBS as negative control. Both groups were injected every another 2 days. After 3 weeks, all the mice were sacrificed and tumor lesions were excised and photographed. The tumor volume $\left(\mathrm{mm}^{3}\right)$ was calculated as length $\times$ width $^{2} / 2$. All the mice were purchased from Vital River Laboratories (China), and kept in SPF environment at the Experimental Animal Center of China Medical University, which was approved by China Medical University Animal Care and Use Committee and complied with national animal experimental criteria.

\section{Dual-luciferase reporter assay}

The binding sequences of miR-1182 and miR-145 on circWHSC1were cloned into pSI-check Dual-luciferase vectors (Hanbio Biotechnology, China). HEK-293 T cells were co-transfected with miR-1182 and miR-145 mimics and the wild type or mutated dual luciferase plamid. The cells were harvested at $48 \mathrm{~h}$ after transfection, and the luciferase activities were measured with Dual-Luciferase Reporter Assay System (Promega, USA). The relative luciferase intensity was normalized to renilla luciferase activity.

\section{RNA pull-down assay}

BeaverBeads ${ }^{\text {th }}$ Streptavidin (BEAVER, SuZhou, China) was used to capture the biotin-labeled circWHSC1 probe, the empty vector was used as a control. The biotinylated nucleic acid compound was incubated with protein lysates of ovarian cancer cells OVCAR3 and CAOV3 cells overnight at $4{ }^{\circ} \mathrm{C}$ on the shaker, to specifically capture circWHSC1 and the corresponding conjugate. After elution of the magnetic beads, the bound RNA was extracted with TRIzol reagent, and the expression of miR-1182 and miR-145 were detected by qRT-PCR. The sequences of the probe against circWHSC1 can be found in Additional file 1: Table S3.

\section{Isolation of exosomes from cell culture medium}

The cells were cultured in Exo-Clear ${ }^{\mathrm{rm}}$ cell growth medium (SBI, USA) for $36 \mathrm{~h}$ before extracting the supernatant. The $0.22 \mu \mathrm{m}$ Millipore Express ${ }^{\circ}$ PES Membrane (Millex, USA) was used to filter dead cells in supernatant. After $30 \mathrm{~min}$ of concentration at $10,000 \times \mathrm{g}$, the supernatant was ultracentrifugated at $100,000 \times \mathrm{g}$ for 70 min (Optima XPN-100 Ultracentrifuge, Beckman, USA), and the pellet containing exosomes was collected. The pellet was washed in PBS and then concentrated at 100, $000 \times \mathrm{g}$ for another $70 \mathrm{~min}$.

\section{Transmission electron microscopy}

After ultracentrifugation, $2 \mu \mathrm{l}$ of exosome pellet was dripped onto a carbon-coated copper grid and dried at $37^{\circ} \mathrm{C}$ for $30 \mathrm{~min} .20 \mathrm{~g} / \mathrm{L}$ phosphotungstic acid was used to stained exosomes for $3 \mathrm{~min}$. The morphology of exosomes was photographed by $\mathrm{H}-7650$ transmission electron microscope.

\section{Cell morphology monitor}

Before treated with exosomes, HMrSV5 cells were starved in serum-free medium for $12 \mathrm{~h}$. After $24 \mathrm{~h}$ of coculture with exosomal circWHSC1, which was extracted from circWHSC1-overexpressing cells conditioned culture medium, HMrSV5 cells were photographed.

\section{HE staining}

The peritoneum of nude mice were excised and fixed in paraformaldehyde. After embedding and paraffin sectioning, the slices were dewaxed with xylene, dehydrated with gradient alcohol, and then stained with hematoxylin and eosin. The slices were photographed with Olympus light microscope $(\times 20)$.

\section{Statistics}

All the data were collected at least three independent experiments and presented as mean $\pm \mathrm{SD}$. Two-tailed Student's t-test and $P<0.05$ were used to analyze statistical significance in SPSS 18.0 software.

\section{Results \\ Correlation of circWHSC1 expression with epithelial ovarian carcinoma}

The expression level of circWHSC1 was determined with qRT-PCR. CircWHSC1 was significantly higher expressed in ovarian cancer tissues than that in normal ovaries (Fig. 1a, $p<0.05$ ). Besides, circWHSC1 expression was positively correlated with differentiation (Fig. 1b, Well vs. Moderate and Poor, $p<0.05$, details can be found in Additional file 1: Tables S1, S2).

\section{CircWHSC1 promotes cell viability, migration and invasion ability in ovarian cancer}

QRT-PCR indicated that expression level of circWHSC1 was highest in OVCAR3, and lowest in CAOV3 (Fig. 1c). After transfection of pHBLV-CMV-circWHSC1 plasmid, circWHSC1 was highly expressed in CAOV3 (Fig. 1d, $p<$ 0.05). After sh-circWHSC1-GFP transfection, circWHSC1 was significantly down-regulated in OVCAR3 (Fig. 1e, $p<$ 0.05). Compared with the group transfected with control vector, overexpression of circWHSC1 increased cell growth (Fig. 2a, $p<0.05$ ), reduced cell apoptosis (Fig. 2c, $p<0.05$ ), promoted cell migration (Fig. 2e, $p<0.05$ ) and invasion rate (Fig. $2 \mathrm{~g}, p<0.05$ ). Downregulation of circWHSC1 in OVCAR3 exhibited the opposite effects (Fig. 2b, d, f, h, $p<0.05$ ). 


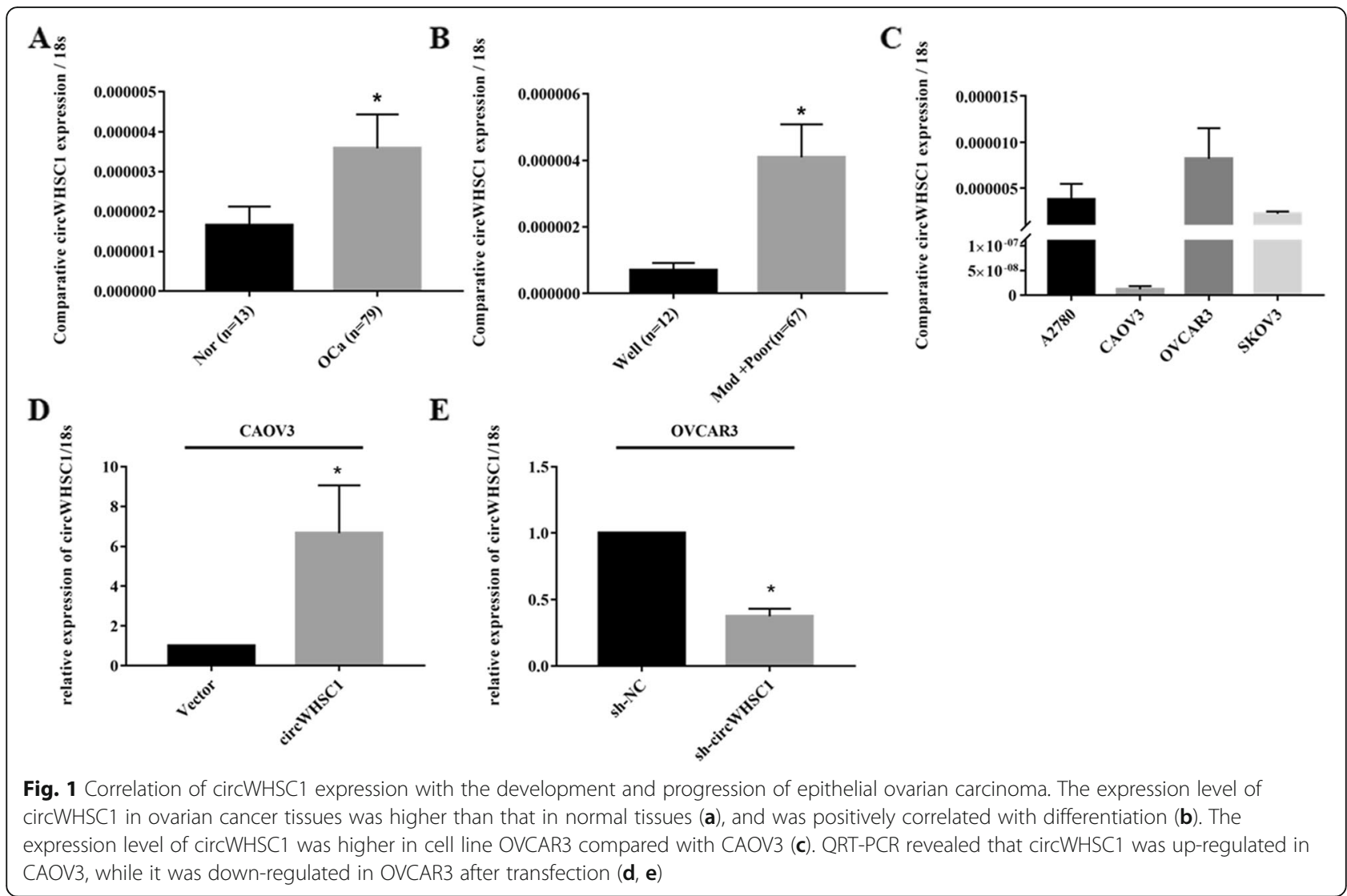

\section{Overexpression of circWHSC1 promotes tumor growth in vivo}

Compared with vector transfected group, circWHSC1overexpressed cells showed a more rapid rate of growth and the tumor volume was larger in nude mice (Fig. 3a, b, $p<$ 0.05). And as shown in Fig. 3c, compared to control group, the expression of circWHSC1 was significantly increased in subcutaneous xenograft of circWHSC1-overexpressed tumor cells $(p<0.05)$.

\section{CircWHSC1 sponges miR-1182 and miR-145}

Based on a circRNA/miRNA interaction prediction website (https://circinteractome.nia.nih.gov/), we predicted that there were complementary sequences with miR-1182 and miR-145 on circWHSC1 (Fig. 4a). Dual-luciferase reporter assay suggested that either miR-1182 or miR-145 could significantly reduce the relative luciferase activity of the wild-type of circWHSC1 luciferase plamid compared with the mutant-type (Fig. 4b, $p<0.05$ ). RNA pull-down assay demonstrated that circWHSC1 binds directly to miR-1182 and miR-145. RNA from RNA pull-down assay with a probe against circWHSC1 was used for qPCR analysis, which demonstrated an enrichment of circWHSC1 and miR-1182 and miR-145 in OVCAR3 and CAOV3. (Fig. 4c, $p<0.05$ ).

\section{CircWHSC1 regulates protein expression of TERT and MUC1}

After overexpression of circWHSC1 in CAOV3, the expression levels of TERT and MUC1 were increased. Down-regulation of circWHSC1 yielded the opposite effects in OVCAR3 (Fig. 4d). TERT and MUC1 were also up-regulated in circWHSC1-overexpression xenograft (Fig. 4e).

Exosomal circWHSC1 promotes peritoneal dissemination and regulates expression of MUC1 in peritoneum

Western blot showed exosome specific markers, such as CD63, CD9, HSP70 and TSG101 were expressed in the extracted exosome pellets (Fig. 5a). Electron microscopic indicated the diameters of the extracted exosomes ranged from 10 to $100 \mathrm{~nm}$ (Fig. 5b).

The morphology of HMrSV5 cells incubated with exosomal circWHSC1 was converted into fibroblast-like (Fig. 5c). After incubation of exosomal circWHSC1, N-cadherin and MUC1 were up-regulated, while E-cadherin was downregulated in HMrSV5 cells (Fig. 5d). To explore the role of exosomal circWHSC1 in tumor peritoneal dissemination, nude mice were intraperitoneal injected with CAOV3 cells for tumorigenesis, and then injected with circWHSC1 exosomes or PBS as control group every 2 days. For the group treated with exosomes, the number of tumor nodules was 


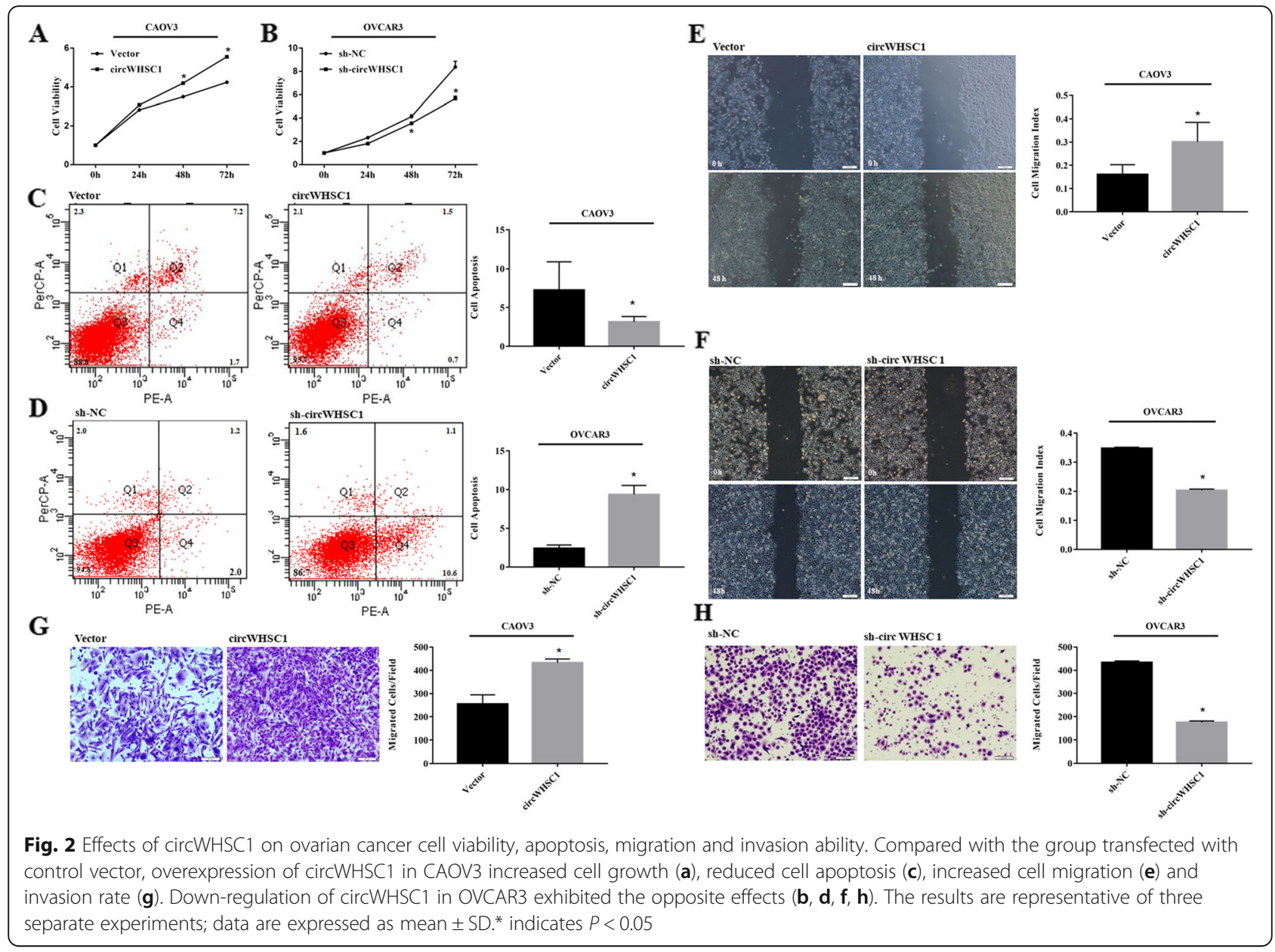

significantly increased in abdominal cavity (Fig. 5e). HE staining showed that the peritoneum was covered with a monolayer of mesothelial cells with intact intercellular junctions (Fig. 5f). After the treatment of exosomal circWHSC1, mesothelial cells arranged loosely, and the stromal layer was reactively thicken surrounding infiltration of tumor cells (Fig. 5g).

\section{Discussion}

In this study, we used circRNA microarray to screen for the differentially expressed circRNAs and discovered that hsa_circ_0001387 (http://www.circbase.org/) was highly expressed in ovarian cancer compared with normal ovaries. Furthermore, we used RT-qPCR to detect and quantify its expression level in 79 cases of epithelial ovarian carcinoma and 13 cases of normal ovarian tissues. The results showed that circWHSC1 expression level was significantly higher in ovarian carcinoma than normal ovaries, and was positively correlated with differentiation, which revealed that circWHSC1 might correlate with the development of ovarian carcinoma.
We performed cell function assays to verify circWHSC1's functions in ovarian cancer. The specific shRNA targeting the splicing sites was used to destroy the circular structure of circWHSC1 in OVCAR3. Downregulation of circWHSC1 suppressed cell proliferation, migration and invasion, and induced cell apoptosis. Overexpression of circWHSC1 in CAOV3 exerted the opposite effects. Compared with the control group, subcutaneous xenograft of circWHSC1-overexpressed tumor cells resulted in an increase in tumor formation ability and its volume in nude mice. These results indicated the highly expressed circWHSC1 plays an important role in tumorigenesis and development of ovarian cancer.

It has been reported that circRNA can adsorb microRNA and release its inhibitory effect on downstream targets [4]. Through competing endogenous RNA (ceRNA) network, circRNA regulates the expression of cancer-related genes and participates in tumorigenesis $[6,7]$. Based on this theory, miR-145 and miR-1182 were found to contain complementary sequences with circWHSC1 according to bioinformatics prediction (https://circinteractome.nia.nih.gov/), which was further confirmed by dual-luciferase reporter assay and RNA pull-down assay in our study. 


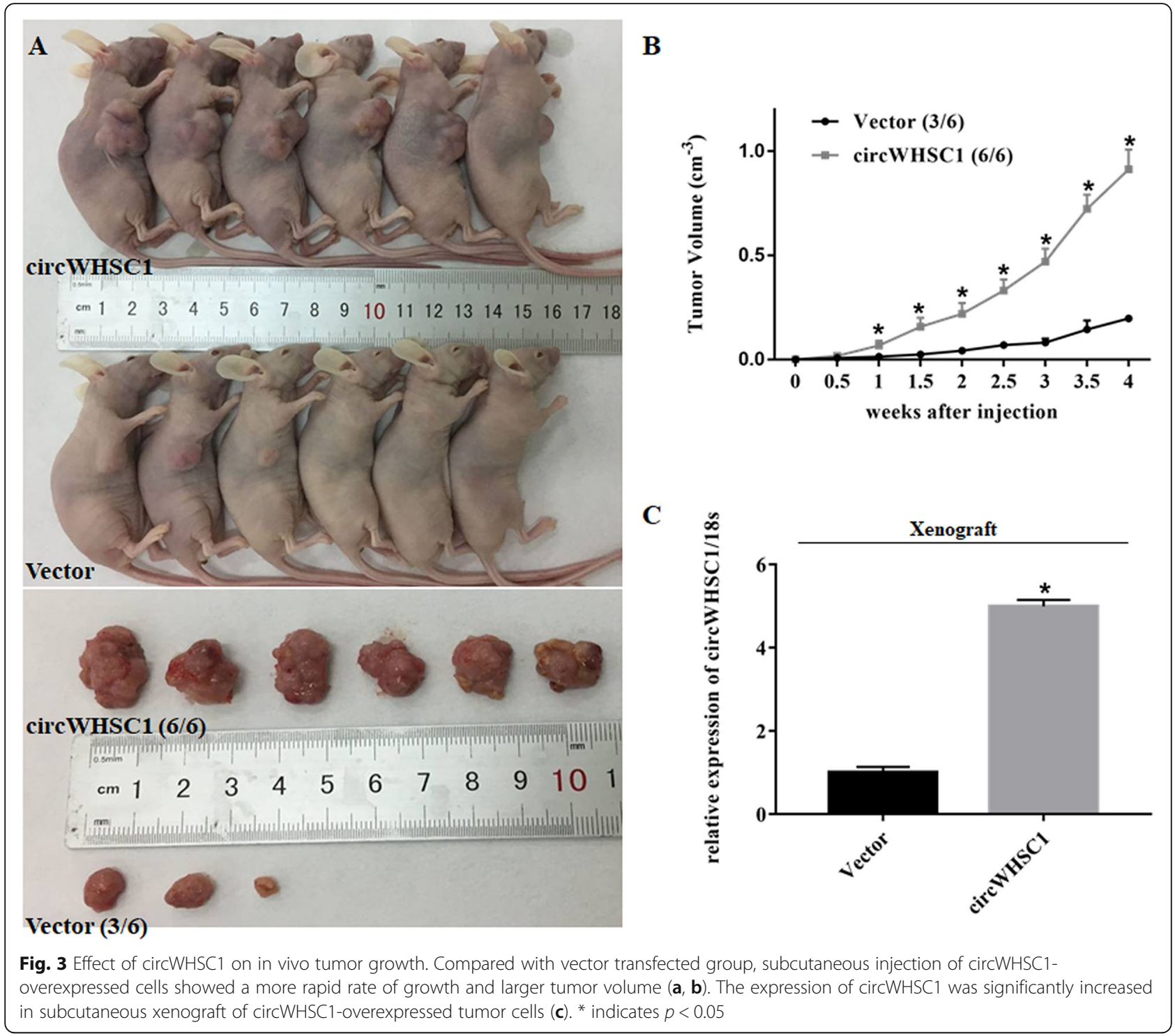

According to Hou et al. [8], up-regulation of miR-1182 suppressed cell proliferation, invasion ability, and decreased the expression of hTERT in ovarian cancer. TERT has been confirmed as a direct target of miR-1182 by luciferase assays. Similar effects could be found in gastric cancer [9] and bladder cancer [10]. In addition, miR-145 is down-regulated in ovarian cancer tissues and acts as a tumor suppressor. MiR-145 overexpression leads to the inhibition of colony formation, cell proliferation, cell growth viability and invasion, and the induction of cell apoptosis through directly targeting upregulation of $\mathrm{MUCl}$, which is also verified by luciferase report assays [11]. In our study, western blot assay showed that the expression levels of hTERT and MUC1 were increased in cells after up-regulation of circWHSC1, while down-regulation of circWHSC1 decreased the expression of both targets. Therefore, we concluded that circWHSC1 could affect the progression of ovarian cancer by sponging miR-1182 and miR-145, and regulated the expression of these two oncogenes.

Human telomerase reverse transcriptase (hTERT) acts as the major catalytic component of telomerase and regulates the extension of telomeres [12, 13]. TERT has been reported to be activated in cancer cell lines and plays an important role in growth, migration and invasion of cancer cells $[14,15]$. In ovarian cancer cells, ectopic expression of TERT could induce epithelial-to-mesenchymal transition (EMT) by up-regulating of Slug [16, 17].

MUC1 (mucin 1) is a well-characterized member of membrane-bound mucins, modulates cell-to-cell and cell-extracellular matrix interactions and participates in cancer cell behaviors $[18,19]$. As an oncogene, 


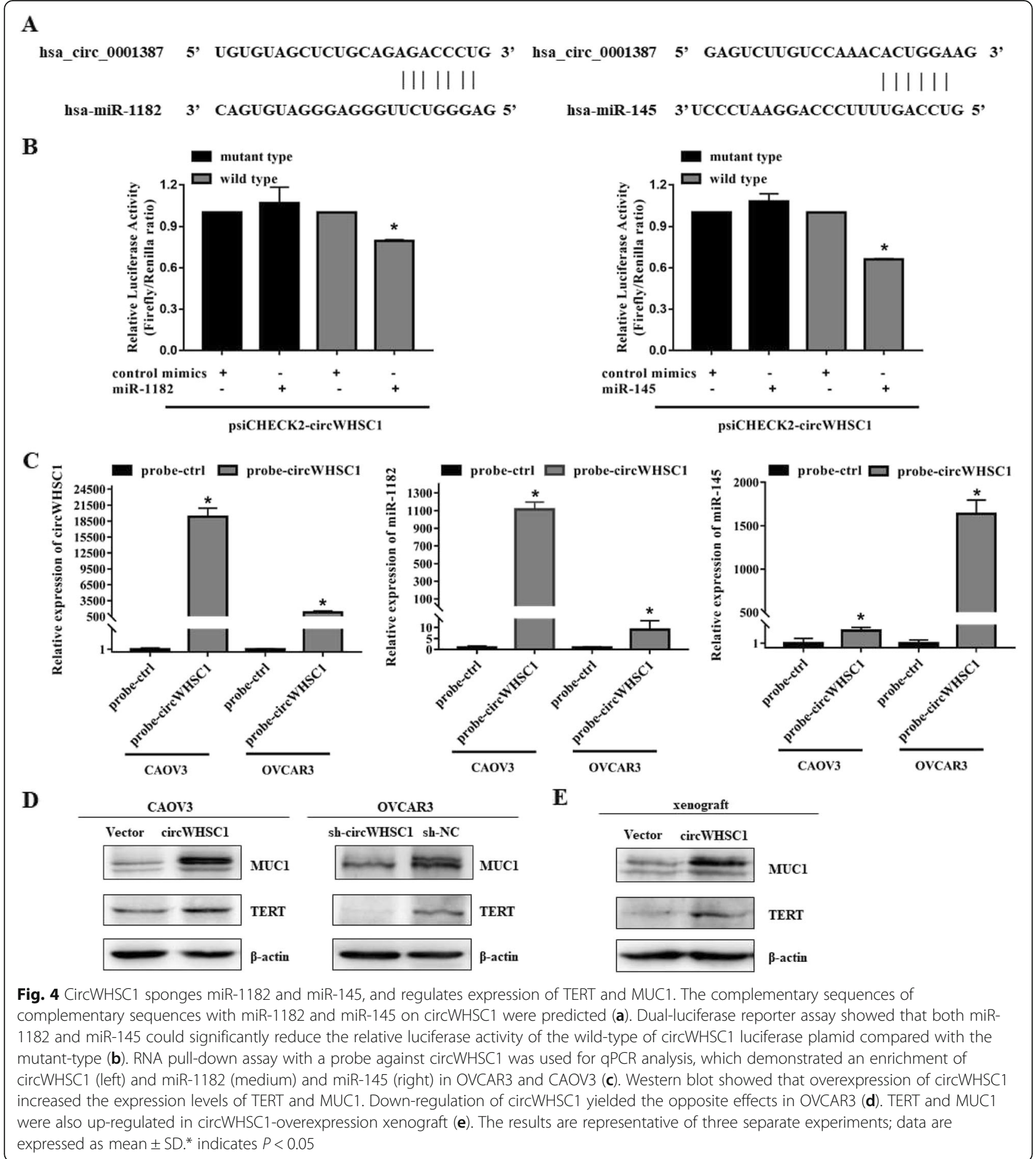

MUC1 is highly expressed in a variety of human adenocarcinomas, including pancreatic cancer [20], non-small cell lung cancer [21], gastric cancer [22] and ovarian cancer [23]. MUC1 coordinates invasive growth of tumor cells [24], triggers EMT process and participates in cancer metastasis, which is associated with poor prognosis [20-22]. In ovarian cancer, MUC1 is highly expressed compared to normal tissues and participates in cellular transformation and tumorigenicity [23].

In addition, Cho et al. [25] found that detecting the expression level of MUC1 in ascites can help distinguish 


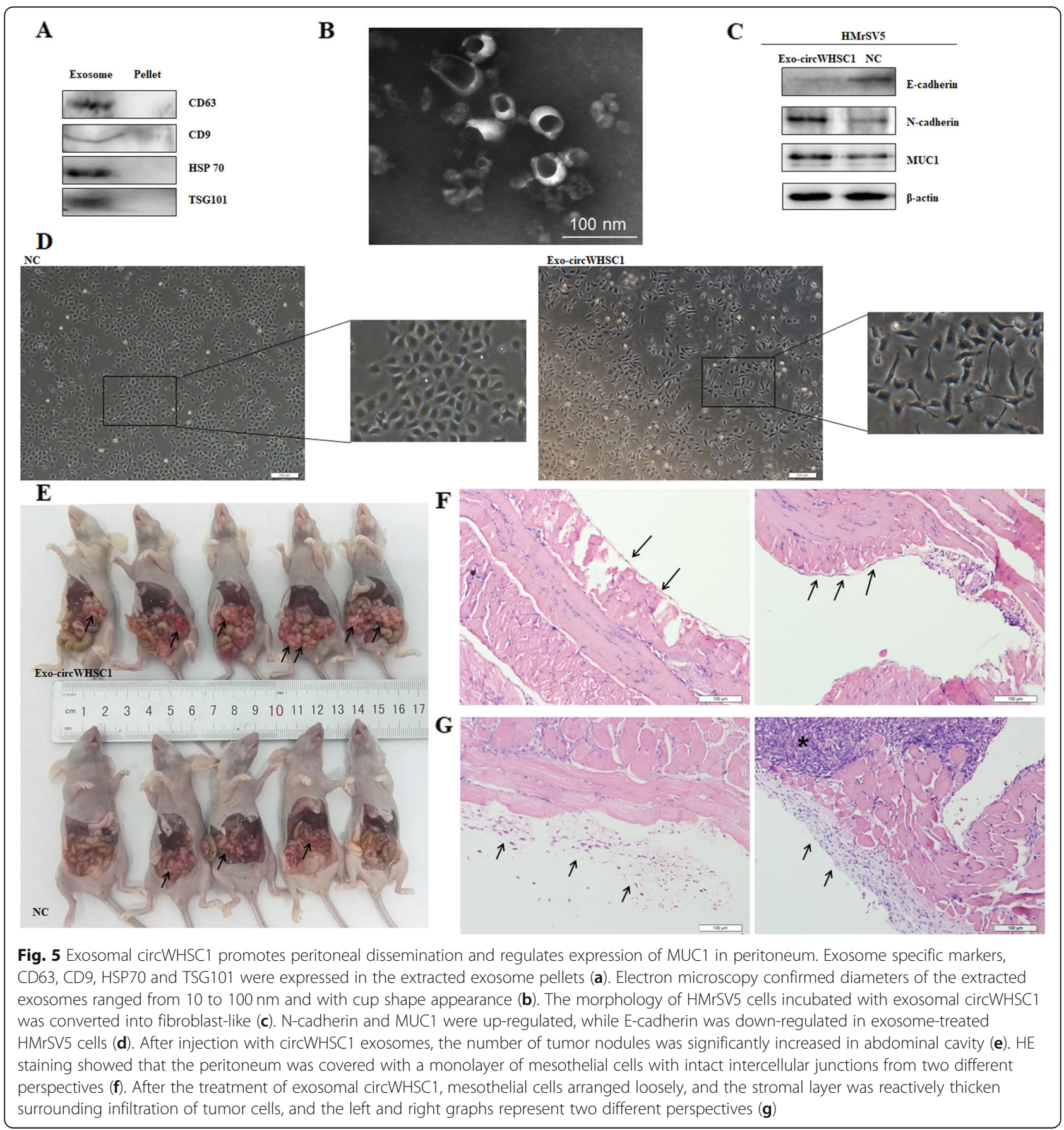

between metastatic adenocarcinoma cells and reactive mesothelial cells, with a sensitivity of up to $99 \%$ and certain diagnostic value. Similar studies have reported that MUC1 immunoreactivity can be detected in ascites samples from patients with ovarian serous carcinoma and pancreatic cancer [26]. Moreover, compared with benign mesothelial cells, MUC1 was significantly higher in malignant mesothelioma [27]. Therefore, we wonder whether circWHSC1 can be secreted by ovarian cancer cells in the form of exosomes, taken up by peritoneal mesothelial cells, inducing up-regulation of MUC1 expression in mesothelial cells, and transformed into malignant mesothelial cells, which favors for peritoneal dissmination and tumor implantation.

Exosomes are small extracellular vesicles, function as vehicles of active protein and RNA, thus mediate cell-tocell communications and regulates biological behavior of recipient cells [28-34]. We used PCR to confirm that 
circWHSC1 can be secreted into exosomes, furthermore, western blot showed exosome specific markers, such as CD63, CD9, HSP70 and TSG101 were expressed in the extracted exosome pellets and what's more, electron microscopic indicated the diameters of the extracted exosomes ranged from 10 to $100 \mathrm{~nm}$. The morphology of HMrSV5 cells treated with exo-circWHSC1 was fusiformlike, and the tight junctions between the cells were reduced, while the expression of E-cadherin was decreased, the expression of $\mathrm{N}$-cadherin was increased, which indicated MMT process occurred in mesothelial cells. Similar with epithelial cells gain fibroblast-like phenotype and motility in EMT, MMT (mesothelial-to-mesenchymal transition) refers to mesothelial cells lining the peritoneal cavity convert into carcinoma-associated fibroblasts [35-37]. MMT dissociates intercellular junctions and increases cell adhesion and invasion through the peritoneum, which provides a suitable tumor environment for peritoneal implantation and dissemination [38, 39].

Further, we injected tumor cells into the abdominal cavity of nude mice, and the experimental group was given exo-circWHSC1 continuous stimulation. The results showed that the abdominal circumference of the nude mice increased, the abdominal cavity was transplanted, and HE staining showed that the peritoneal mesothelum lost its original tight structure between cells. In addition, western blotting showed that under exo-circWHSC1 stimulation, the peritoneal mesothelial cells expressed higher level of MUC1, which again verified the "microRNA sponge" effects of circWHSC1 in its receptor cells. Therefore, we put forward that circWHSC1 can be taken up by peritoneal mesothelial cells in the form of exosomes, and induces its EMT changes. Meanwhile, MUC1 is highly expressed, the adhesion of tumor cell to peritoneum and peritoneal dissemination are promoted.

\section{Conclusions}

In summary, we found that circWHSC1, which is highly expressed in ovarian cancer, can adsorb miR-145 and miR-1182, up-regulates the expression of downstream targets MUC1 and hTERT, promotes cancer cell proliferation and invasion, also can be secreted into exosomes. In addition, peritoneal mesothelial cells can act as recipient cells and take up circWHSC1-rich exosomes. This process gives a favorable condition for tumor metastasis, which helps the tumor to spread in peritoneal cavity and promotes the progression of ovarian cancer. Our discovery enriches the research of the molecular biological mechanism of circRNA involved in the development of ovarian cancer, and provides novel ideas for new diagnostic and therapeutic strategies for clinical cancer therapy.

\section{Supplementary information}

Supplementary information accompanies this paper at https://doi.org/10. 1186/s13046-019-1437-z.

Additional file 1. The expression level and sequences of circWHSC1.

Abbreviations

circRNA: Circular RNA; hTERT: Human telomerase reverse transcriptase; miRNA: MicroRNA; MUC1: Mucin 1; qRT-PCR: Real-time quantitative PCR

\begin{abstract}
Acknowledgements
None.

Authors' contributions

$Y Z$ designed the research; $\mathrm{ZH} \mathrm{Z}$ conducted the experiments and wrote the paper; YP D, GX and SC performed the experiments and analyzed the data. All authors read and approved the final manuscript.
\end{abstract}

\section{Funding}

This study was supported by National Natural Scientific Foundation of China (Nos. 81772785; 81772776; 81872115).

\section{Availability of data and materials}

The datasets used and/or analyzed during the present study are available from the corresponding author for reasonable request.

\section{Ethics approval and consent to participate}

All the enrolled patients signed the inform consent, and this research ethics protocol was approved by the China Medical University Ethics Committee (No. 2014-27).

\section{Consent for publication \\ Not applicable.}

\section{Competing interests}

None.

\section{Author details}

${ }^{1}$ Department of Gynecologic Oncology Research Office, The Third Affiliated Hospital of Guangzhou Medical University, No.63 Duobao Road, Liwan District, Guangzhou 510150, Guangdong, China. ${ }^{2}$ Department of Obstetrics and Gynecology, Key Laboratory for Major Obstetric Diseases of Guangdong Province, and Key Laboratory of Reproduction and Genetics of Guangdong Higher Education Institute in Guangdong Province, The Third Affiliated Hospital of Guangzhou Medical University, Guangzhou 510150, China.

${ }^{3}$ Department of Gynecology, the First Affiliated Hospital of China Medical University, Shenyang 110001, China.

Received: 26 May 2019 Accepted: 2 October 2019

Published online: 30 October 2019

\section{References}

1. Siegel RL, Miller KD, Jemal A. Cancer statistics, 2018. CA Cancer J Clin. 2018; 68:7-30.

2. Torre LA, Trabert B, DeSantis CE, Miller KD, Samimi G, Runowicz CD, et al. Ovarian cancer statistics, 2018. CA Cancer J Clin. 2018;68(4):284-96.

3. Hansen TB, Jensen TI, Clausen BH, Bramsen JB, Finsen B, Damgaard CK, et al. Natural RNA circles function as efficient microRNA sponges. Nature. 2013; 495:384-8.

4. Zhang $Y$, Zhang $X O$, Chen T, Xiang JF, Yin QF, Xing YH, et al. Circular intronic long noncoding RNAs. Mol Cell. 2013;51(6):792-806.

5. Li Y, Zheng Q, Bao C, Li S, Guo W, Zhao J, et al. Circular RNA is enriched and stable in exosomes: a promising biomarker for cancer diagnosis. Cell Res. 2015;25(8):981-4.

6. Zheng Q, Bao C, Guo W, Li S, Chen J, Chen B, et al. Circular RNA profiling reveals an abundant circHIPK3 that regulates cell growth by sponging multiple miRNAs. Nat Commun. 2016:7:11215.

7. Hsiao KY, Lin YC, Gupta SK, Chang N, Yen L, Sun HS, et al. Noncoding effects of circular RNA CCDC66 promote Colon Cancer growth and metastasis. Cancer Res. 2017;77(9):2339-50. 
8. Hou XS, Han CQ, Zhang W. MiR-1182 inhibited metastasis and proliferation of ovarian cancer by targeting hTERT. Eur Rev Med Pharmacol Sci. 2018; 22(6):1622-8.

9. Zhang D, Xiao YF, Zhang JW, Xie R, Hu CJ, Tang B, et al. miR-1182 attenuates gastric cancer proliferation and metastasis by targeting the open reading frame of hTERT. Cancer Lett. 2015;360(2):151-9.

10. Zhou J, Dai W, Song J. miR-1182 inhibits growth and mediates the chemosensitivity of bladder cancer by targeting hTERT. Biochem Biophys Res Commun. 2016;470(2):445-52

11. Wu H, Xiao Z, Wang K, Liu W, Hao Q. MiR-145 is downregulated in human ovarian cancer and modulates cell growth and invasion by targeting p70S6K1 and MUC1. Biochem Biophys Res Commun. 2013;441(4):693-700.

12. Yi X, Tesmer VM, Savre-Train I, Shay JW, Wright WE. Both transcriptional and posttranscriptional mechanisms regulate human telomerase template RNA levels. Mol Cell Biol. 1999;19(6):3989-97.

13. Cong YS, Wright WE, Shay JW. Human telomerase and its regulation. Microbiol Mol Biol Rev. 2002;66(3):407-25.

14. Bernardes de Jesus B, Blasco MA. Telomerase at the intersection of cancer and aging. Trends Genet. 2013;29(9):513-20.

15. Takakura M, Kyo S, Kanaya T, Hirano H, Takeda J, Yutsudo M, et al. Cloning of human telomerase catalytic subunit (hTERT) gene promoter and identification of proximal core promoter sequences essential for transcriptional activation in immortalized and cancer cells. Cancer Res. 1999. 59(3):551-7.

16. Choi MJ, Cho KH, Lee S, Bae YJ, Jeong KJ, Rha SY, et al. hTERT mediates norepinephrine-induced slug expression and ovarian cancer aggressiveness. Oncogene. 2015;34(26):3402-12.

17. Kuwabara Y, Yamada T, Yamazaki K, Du WL, Banno K, Aoki D, et al. Establishment of an ovarian metastasis model and possible involvement of E-cadherin down-regulation in the metastasis. Cancer Sci. 2008;99:1933-9.

18. Jonckheere $N$, Van Seuningen I. The membrane-bound mucins: how large O-glycoproteins play key roles in epithelial cancers and hold promise as biological tools for gene-based and immunotherapies. Crit Rev Oncog. 2008;14(2-3):177-96.

19. Jonckheere N, Van Seuningen I. The membrane-bound mucins: from cell signalling to transcriptional regulation and expression in epithelial cancers. Biochimie. 2010;92:1-11.

20. Roy LD, Sahraei M, Subramani DB, Besmer D, Nath S, Tinder TL, et al. MUC enhances invasiveness of pancreatic cancer cells by inducing epithelial to mesenchymal transition. Oncogene. 2011;30(12):1449-59.

21. Hirasawa Y, Kohno N, Yokoyama A, Kondo K, Hiwada K, Miyake M. Natural autoantibody to MUC1 is a prognostic indicator for non-small cell lung cancer. Am J Respir Crit Care Med. 2000;161 (2 Pt 1):589-94.

22. Ohno T, Aihara R, Kamiyama Y, Mochiki E, Asao T, Kuwano H. Prognostic significance of combined expression of MUC1 and adhesion molecules in advanced gastric cancer. Eur J Cancer. 2006;42(2):256-63.

23. Deng J, Wang L, Chen H, Li L, Ma Y, Ni J, et al. The role of tumourassociated MUC1 in epithelial ovarian cancer metastasis and progression. Cancer Metastasis Rev. 2013;32(3-4):535-51.

24. Ye Q, Yan Z, Liao X, Li Y, Yang J, Sun J, et al. MUC1 induces metastasis in esophageal squamous cell carcinoma by upregulating matrix metalloproteinase 13. Lab Investig. 2011;91(5):778-87.

25. Cho JS, Kim GE, Lee JS, Lee JH, Nam JH, Choi C. Diagnostic usefulness of MUC1 and MUC4 for distinguishing between metastatic adenocarcinoma cells and reactive mesothelial cells in effusion cell blocks. Acta Cytol. 2013; 57(4):377-83.

26. Han L, Pansare V, Al-Abbadi M, Husain M, Feng J. Combination of MUC5ac and WT-1 immunohistochemistry is useful in distinguishing pancreatic ductal carcinoma from ovarian serous carcinoma in effusion cytology. Diagn Cytopathol. 2010;38(5):333-6.

27. Creaney J, Segal A, Sterrett G, Platten MA, Baker E, Murch AR, Nowak AK, Robinson BW, Millward MJ. Overexpression and altered glycosylation of MUC1 in malignant mesothelioma. Br J Cancer. 2008;98(9):1562-9.

28. Akers JC, Gonda D, Kim R, Carter BS, Chen CC. Biogenesis of extracellular vesicles (EV): exosomes, microvesicles, retrovirus-like vesicles, and apoptotic bodies. J Neuro-Oncol. 2013;113(1):1-11.

29. Liu Y, Gu Y, Han Y, Zhang Q, Jiang Z, Zhang X, et al. Tumor Exosomal RNAs promote lung pre-metastatic niche formation by activating alveolar epithelial TLR3 to recruit neutrophils. Cancer Cell. 2016;30(2):243-56.

30. Tokuhisa M, Ichikawa Y, Kosaka N, Ochiya T, Yashiro M, Hirakawa K, et al. Exosomal miRNAs from peritoneum lavage fluid as potential prognostic biomarkers of peritoneal metastasis in gastric Cancer. PLoS One. 2015;10(7): e0130472.

31. Cizmar P, Yuana Y. Detection and characterization of extracellular vesicles byTransmission and Cryo-transmission Electron microscopy. Methods Mol Biol. 2017;1660:221-32

32. Lötvall J, Hill AF, Hochberg F, Buzás El, Di Vizio D, Gardiner C, et al. Minimal experimental requirements for definition of extracellular vesicles and their functions: a position statement from the International Society for Extracellular Vesicles. J Extracell Vesicles. 2014;3:26913.

33. Taylor DD, Gercel-Taylor C. Exosomes/microvesicles: mediators of cancerassociated immunosuppressive microenvironments. Seminlmmunopathol. 2011;33(5):441-54

34. Mathivanan S, Simpson RJ. ExoCarta: a compendium of exosomal proteins and RNA. Proteomics. 2009;9(21):4997-5000.

35. Birchmeier W, Behrens J. Cadherin expression in carcinomas: role in the formation of cell junctions and the prevention of invasiveness. Biochim Biophys Acta. 1994;1198:11-26.

36. Sawada K, Mitra AK, Radjabi AR, Bhaskar V, Kistner EO, Tretiakova M, et al. Loss of $\mathrm{E}$-cadherin promotes ovarian cancer metastasis via a5-integrin, which is a therapeutic target. Cancer Res. 2008;68:2329-39.

37. Sandoval P, Jiménez-Heffernan JA, Rynne-Vidal Á, Pérez-Lozano ML, Gilsanz A, Ruiz-Carpio V, et al. Carcinoma-associated fibroblasts derive from mesothelial cells via mesothelial-to-mesenchymal transition in peritoneal metastasis. J Pathol. 2013;231(4):517-31.

38. Rynne-Vidal A, Au-Yeung CL, Jiménez-Heffernan JA, Pérez-Lozano ML, Cremades-Jimeno L, Bárcena C, et al. Mesothelial-to-mesenchymal transition as a possible therapeutic target in peritoneal metastasis of ovarian cancer. J Pathol. 2017;242(2):140-51.

39. Nakamura M, Ono YJ, Kanemura M, Tanaka T, Hayashi M, Terai Y, et al. Hepatocyte growth factor secreted by ovarian cancer cells stimulates peritoneal implantation via the mesothelial-mesenchymal transition of the peritoneum. Gynecol Oncol. 2015;139(2):345-54.

\section{Publisher's Note}

Springer Nature remains neutral with regard to jurisdictional claims in published maps and institutional affiliations.

Ready to submit your research? Choose BMC and benefit from

- fast, convenient online submission

- thorough peer review by experienced researchers in your field

- rapid publication on acceptance

- support for research data, including large and complex data types

- gold Open Access which fosters wider collaboration and increased citations

- maximum visibility for your research: over $100 \mathrm{M}$ website views per year

At BMC, research is always in progress.

Learn more biomedcentral.com/submissions 